\title{
Thermodynamic Properties of Solubility for Nitrophenyl Derivatives of Furfural Oxime in Organic Solvents
}

\author{
Andriy Marshalek ${ }^{1 *}$, Iryna Sobechko ${ }^{1}$, Yuriy Horak ${ }^{2}$, Volodymyr Dibrivnyi ${ }^{1}$ \\ ${ }^{1}$ Lviv National Polytechnic University, S. Bandery str. 12, Lviv, Ukraine \\ ${ }^{2}$ Lviv National University named after Ivan Franko, Kyryla and Mefodiya str. 6, Lviv, Ukraine \\ Email: yourowndarkness@gmail.com
}

\begin{abstract}
Enthalpies and entropies of dissolution in acetonitrile, propan-2-ol and ethyl acetate were determined from the results of temperature dependence of solubility for 5-(2-nitro phenyl)-2-furyl oxime, 5-(3-nitro phenyl)-2-furyl oxime and 5-(4-nitro phenyl)-2-furyl oxime. Sublimation enthalpies and entropies were determined using integral effusion Knudsen method; adjustment of these values to 298K was conducted. Using these values the thermodynamic properties of solvation in the investigated systems were calculated. The influence of physical properties of the solvent on dissolution of the investigated compounds was analyzed.
\end{abstract}

Keywords: 5-(2-nitro phenyl)-2-furyl oxime, enthalpy, entropy, solubility, sublimation, solvation

\section{Introduction}

Heterocyclic compounds are very common in nature. Most of them take part in biological processes. Phenyl-furanic fragment is present in a lot of substances which develop a wide range of biological activity. Lately, functional compounds with phenyl-furanic fragment are widely used in multi-component reactions that allow optimizing the resource usage and conducing the green chemistry concept. Most of the parameters for the processes of syntheses for these compounds were chosen using the method of probes and mistakes. Determination of thermodynamic properties for compounds with phenyl-furanic fragment allows to calculate energetic properties of their molecules and to decrease the energy usage at separate stages of processes of their synthesis, purification and use. Investigated in the current work furfural oximes are used as soil nitrification inhibitors [1], intermediates in the synthesis of medicines with tuberculostatic [2], antifungal [3] properties, and for many syntheses of disubstituted furan derivatives[4]. Phenyl derivatives of furfural oxime show antispasmodic [5], vasodilator [6], cardiotropic [7] and antiviral [8] properties. Most processes with their participation take place in solvents. That's why thermodynamic properties of furfural oxime and its nitrophenyl derivatives solubility are needed for optimization and calculation of energy balances for processes of synthesis, purification and application of these compounds, which has become the main aim of this work.

\section{$2 \quad$ Experimental}

Structural formulas of 5-(2-nitro phenyl)-2-furyl oxime (A), 5-(3-nitro phenyl)-2-furyl oxime (B) and 5(4-nitro phenyl)-2-furyl oxime (C) are shown in Figure 1.

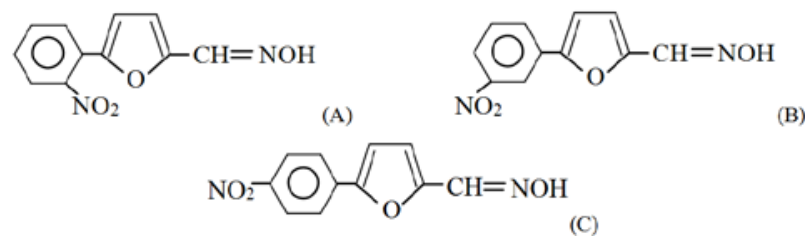

Figure 1. Structural formulas of the investigated compounds 
The synthesis of investigated compounds was conducted by the method, shown in Figure 2.

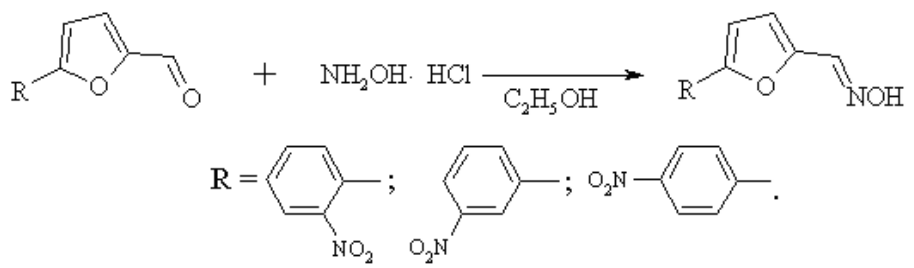

Figure 2. Synthesis of the investigated compounds

Mixture containing $0.023 \mathrm{~mol}$ of 5-(2-nitro phenyl)-2-furyl-carbaldehyde (for A), 5-(3-nitro phenyl)-2furyl-carbaldehyde (for B) or 5-(4-nitro phenyl)-2-furyl-carbaldehyde (for C), 0.03 mol of hydroxylamine hydrochloride and $2 \mathrm{~g}$ of melted sodium acetate in $20 \mathrm{ml}$ of ethanol was boiled during 4 hours. $30 \mathrm{ml}$ of water was added after cooling with mixing. Obtained residue was filtered and recrystallized from ethanol.

The structure of the synthesized compounds was confirmed by NMR-spectroscopy results. ${ }^{1} \mathrm{H}$ NMR spectra were recorded using Varian $600(600 \mathrm{MHz})$ spectrometer. DMSO and acetone were used as solvents. Chemical shifts ( $\delta . \mathrm{ppm})$ are listed relatively to DMSO and acetone signal. ${ }^{1} \mathrm{H}$ NMR spectra data is listed in table 1.

Table 1. ${ }^{1} \mathrm{H}$ NMR spectra of the investigated compounds.

\begin{tabular}{|c|c|}
\hline Compound & Chemical shifts, $\delta$, ppm \\
\hline $\mathrm{A}$ & $\begin{array}{l}{ }^{1} \mathrm{H} \text { NMR }(600 \mathrm{MHz}, \text { Acetone }-d 6) \delta 6.96(\mathrm{~d}, J=3.5 \mathrm{~Hz}, 1 \mathrm{H}, \text { fur }) .7 .41(\mathrm{~d}, J=3.5 \mathrm{~Hz}, 1 \mathrm{H}, \text { fur }) \\
7.47(\mathrm{~s}, 1 \mathrm{H}, \mathrm{CH}), 7.64\left(\mathrm{t}, J=8.7, \mathrm{~Hz}, 1 \mathrm{H}, \mathrm{C}_{6} \mathrm{H}_{3}\right), 7.78\left(\mathrm{t}, J=8.1, \mathrm{~Hz}, 1 \mathrm{H}, \mathrm{C}_{6} \mathrm{H}_{3}\right), 7.88(\mathrm{~d}, J=8.0 \\
\left.\mathrm{Hz}, 1 \mathrm{H}, \mathrm{C}_{6} \mathrm{H}_{3}\right), 7.91\left(\mathrm{~d}, J=7.2 \mathrm{~Hz}, 1 \mathrm{H}, \mathrm{C}_{6} \mathrm{H}_{3}\right), 11.20(\mathrm{~s}, 1 \mathrm{H}, \mathrm{NH}) .\end{array}$ \\
\hline B & $\begin{array}{l}{ }^{1} \mathrm{H} \text { NMR }(600 \mathrm{MHz}, \mathrm{DMSO}) \delta 7.32(\mathrm{~d}, J=3.3 \mathrm{~Hz}, 1 \mathrm{H}, \text { fur }), 7.43(\mathrm{~d}, J=3.3 \mathrm{~Hz}, 1 \mathrm{H}, \text { fur }), 7.68(\mathrm{~s}, \\
1 \mathrm{H}, \mathrm{CH}), 7.74\left(\mathrm{t}, J=8.0 \mathrm{~Hz}, 1 \mathrm{H}, \mathrm{C}_{6} \mathrm{H}_{4}\right), 8.19\left(\mathrm{~d}, J=7.7 \mathrm{~Hz}, 1 \mathrm{H}, \mathrm{C}_{6} \mathrm{H}_{4}\right), 8.24(\mathrm{~d}, J=7.9 \mathrm{~Hz}, 1 \mathrm{H}, \\
\left.\mathrm{C}_{6} \mathrm{H}_{4}\right), 8.53\left(\mathrm{~s}, 1 \mathrm{H}, \mathrm{C}_{6} \mathrm{H}_{4}\right), 8.05(\mathrm{~s}, 1 \mathrm{H}, \mathrm{NOH})\end{array}$ \\
\hline $\mathrm{C}^{1}$ & $\begin{array}{l}{ }^{1} \mathrm{H} \text { NMR }(600 \mathrm{MHz}, \mathrm{DMSO}): \delta 12.10(\mathrm{~s}, 0.18 \mathrm{H}, \text { syn-OH }), 11.56(\mathrm{~s}, 0.82 \mathrm{H}, \text { anti-OH }), 8.30(\mathrm{~m}, 2 \mathrm{H}), \\
8.10(\mathrm{~s}, 0.82 \mathrm{H}, \text { anti-CH}), 8.00\left(\mathrm{~d}, J=8.9 \mathrm{~Hz}, 0.36 \mathrm{H}, \text { syn- } \mathrm{C}_{6} \mathrm{H}_{4}\right), 7.97(\mathrm{~d}, J=8.9 \mathrm{~Hz}, 1.64 \mathrm{H}, \text { anti- } \\
\left.\mathrm{C}_{6} \mathrm{H}_{4}\right), 7.67(\mathrm{~s}, 0.18 \mathrm{H}, \text { syn-CH}), 7.45(\mathrm{~d}, J=3.6 \mathrm{~Hz}, 0.18 \mathrm{H}, \text { syn-fur }), 7.41(\mathrm{~d}, J=3.6 \mathrm{~Hz}, 0.82 \mathrm{H}, \\
\text { anti-fur }), 7.36(\mathrm{~d}, J=3.6 \mathrm{~Hz}, 0.18 \mathrm{H}, \text { syn-fur }), 6.91(\mathrm{~d}, J=3.6 \mathrm{~Hz}, 0.82 \mathrm{H}, \text { anti-fur })\end{array}$ \\
\hline
\end{tabular}

Purity of the compounds was confirmed by the results of chromatography using Agilent 1100 HPLC with diode matrix and mass-selective detector on Zorbax SB-C18 column, 4.6mm $\times 15 \mathrm{~mm}$, eluent A acetonitrile-water with $0.1 \%$ TFA (95:5), no impurities were found. Solvents were purified using fractional distillation with further identification by refraction index (1.3726 for ethyl acetate (ref. 1.3724), 1.3463 for acetonitrile (ref. - 1.3460) and 1.3774 for propan-2-ol (ref. - 1.3776)); using gas-liquid chromatograph "LHM-08D" with thermal conductivity detector the contents of main component were confirmed to be $99.9 \%$ mol.

Dissolution of substances was carried in three-neck flask equipped with a thermometer and stirrer. The substance was kept in solvent at room temperature for two days before the beginning of experiments. Mixing time was 45 minutes at a temperature of experiment, the permanence of which ( \pm $0.1 \mathrm{~K})$ was reached using a water thermostat. The rate of stirring $(50 \mathrm{rpm})$ was selected so that all solid phase was in the suspended state. Sampling was performed after complete precipitation of solid particles. To confirm the balance experiments were conducted in the both modes of temperature ascending and descending. The absence of hysteresis loop on the curve of the temperature dependence of the solubility reaffirmed achieving a state close to equilibrium. The mass of saturated solutions was determined by mass difference of cups before and after sampling weighted on the VLR-20 scales with an accuracy of $0.00005 \mathrm{~g}$. Then the cups were placed in the oven, where the solvent evaporated at 323-333 K until the

\footnotetext{
1 syn:anti conformers correlation is 9:41 $(\sim 1: 4.55)$
} 
cup reached constant weight. The mass of solid residue was determined by weight difference between the cup with sample and the cup before sampling.

Solute mass $\left(m_{2}\right)$, solubility in mol fractions $\left(x_{2}\right)$, and temperature $(T)$ of the experiments are listed in table 2 along with the coefficients of Schroeder's equation, obtained during experimental data processing. Here and hereafter experimental data was processed using the least squares method with Student's coefficient of $95 \%$ level of confidence.

Table 2. Results of temperature dependence of solubility investigation

\begin{tabular}{|c|c|c|c|c|c|c|c|}
\hline № & $m_{2}, g$ & $x_{2}$ & $\mathrm{~T}, \mathrm{~K}$ & № & $m_{2}, g$ & $x_{2}$ & $\mathrm{~T}, \mathrm{~K}$ \\
\hline \multicolumn{8}{|c|}{ 5-(2-nitro phenyl)-furyl-2-oxime } \\
\hline \multicolumn{8}{|c|}{ Propan-2-ol } \\
\hline 1 & 0.02350 & 0.01591 & 308.2 & 18 & 0.03220 & 0.02273 & 319.6 \\
\hline 2 & 0.02280 & 0.01572 & 308.2 & 19 & 0.03365 & 0.02276 & 319.6 \\
\hline 3 & 0.02165 & 0.01589 & 308.2 & 20 & 0.03170 & 0.02273 & 319.6 \\
\hline 4 & 0.02375 & 0.01649 & 309.6 & 21 & 0.03555 & 0.02398 & 321.2 \\
\hline 5 & 0.02415 & 0.01638 & 309.6 & 22 & 0.03370 & 0.02409 & 321.2 \\
\hline 6 & 0.02655 & 0.01748 & 311.5 & 23 & 0.03460 & 0.02385 & 321.2 \\
\hline 7 & 0.02490 & 0.01726 & 311.5 & 24 & 0.03085 & 0.02251 & 319.2 \\
\hline 8 & 0.02565 & 0.01737 & 311.5 & 25 & 0.03355 & 0.02235 & 319.2 \\
\hline 9 & 0.02665 & 0.01849 & 313.4 & 26 & 0.03100 & 0.02208 & 319.2 \\
\hline 10 & 0.02635 & 0.01836 & 313.4 & 27 & 0.01785 & 0.01218 & 299.5 \\
\hline 11 & 0.02590 & 0.01820 & 313.4 & 28 & 0.01850 & 0.01214 & 299.5 \\
\hline 12 & 0.02945 & 0.02025 & 315.9 & 29 & 0.01750 & 0.01238 & 299.5 \\
\hline 13 & 0.02915 & 0.02004 & 315.9 & 30 & 0.01910 & 0.01298 & 302.3 \\
\hline 14 & 0.02815 & 0.01973 & 315.9 & 31 & 0.01880 & 0.01315 & 302.3 \\
\hline 15 & 0.03315 & 0.02167 & 317.6 & 32 & 0.01880 & 0.01286 & 302.3 \\
\hline 16 & 0.03000 & 0.02153 & 317.6 & 33 & 0.02060 & 0.01448 & 305.8 \\
\hline 17 & 0.03050 & 0.02155 & 317.6 & 34 & 0.02130 & 0.01435 & 305.8 \\
\hline \multicolumn{8}{|c|}{$\left.\ln x_{2}=5.75 \pm 0.25-\cdot(3050 \pm 77) / T\right)$} \\
\hline \multicolumn{8}{|c|}{ Acetonitrile } \\
\hline 1 & 0.04545 & 0.02159 & 294.3 & 18 & 0.06060 & 0.02981 & 305.4 \\
\hline 2 & 0.04415 & 0.02108 & 294.3 & 19 & 0.05645 & 0.03205 & 308.5 \\
\hline 3 & 0.04455 & 0.02106 & 294.3 & 20 & 0.06685 & 0.03199 & 308.5 \\
\hline 4 & 0.04615 & 0.02235 & 296.6 & 21 & 0.06590 & 0.03202 & 308.5 \\
\hline 5 & 0.04710 & 0.02236 & 296.6 & 22 & 0.06975 & 0.03460 & 310.6 \\
\hline 6 & 0.04845 & 0.02216 & 296.6 & 23 & 0.06755 & 0.03432 & 310.6 \\
\hline 7 & 0.04655 & 0.02336 & 297.9 & 24 & 0.07090 & 0.03432 & 310.6 \\
\hline 8 & 0.04895 & 0.02355 & 297.9 & 25 & 0.07460 & 0.03670 & 312.2 \\
\hline 9 & 0.04955 & 0.02329 & 297.9 & 26 & 0.07520 & 0.03667 & 312.2 \\
\hline 10 & 0.04735 & 0.02514 & 300.6 & 27 & 0.07040 & 0.03664 & 312.2 \\
\hline 11 & 0.05190 & 0.02533 & 300.6 & 28 & 0.04545 & 0.02193 & 295.1 \\
\hline 12 & 0.04725 & 0.02503 & 300.6 & 29 & 0.04855 & 0.02201 & 295.1 \\
\hline 13 & 0.05265 & 0.02752 & 302.7 & 30 & 0.04405 & 0.02216 & 295.1 \\
\hline 14 & 0.05345 & 0.02796 & 302.7 & 31 & 0.04555 & 0.02547 & 301.1 \\
\hline 15 & 0.05125 & 0.02726 & 302.7 & 32 & 0.06235 & 0.02514 & 301.1 \\
\hline 16 & 0.06360 & 0.03072 & 305.4 & 33 & 0.05205 & 0.02504 & 301.1 \\
\hline 17 & 0.06120 & 0.02997 & 305.4 & - & - & - & - \\
\hline \multicolumn{8}{|c|}{$\left.\ln x_{2}=5.61 \pm 0.34-\cdot(2789 \pm 102) / T\right)$} \\
\hline \multicolumn{8}{|c|}{ Ethyl acetate } \\
\hline 1 & 0.05610 & 0.05202 & 293.5 & 16 & 0.07720 & 0.06615 & 303.6 \\
\hline
\end{tabular}




\begin{tabular}{|c|c|c|c|c|c|c|c|}
\hline 2 & 0.06010 & 0.05234 & 293.5 & 17 & 0.06885 & 0.06709 & 303.6 \\
\hline 3 & 0.05510 & 0.05198 & 293.5 & 18 & 0.06480 & 0.06851 & 305.8 \\
\hline 4 & 0.05935 & 0.05384 & 295.0 & 19 & 0.08165 & 0.06827 & 305.8 \\
\hline 5 & 0.06370 & 0.05433 & 295.0 & 20 & 0.07040 & 0.06827 & 305.8 \\
\hline 6 & 0.05690 & 0.05455 & 295.0 & 21 & 0.07295 & 0.07129 & 307.9 \\
\hline 7 & 0.06835 & 0.05621 & 297.1 & 22 & 0.07675 & 0.07202 & 307.9 \\
\hline 8 & 0.06390 & 0.05629 & 297.1 & 23 & 0.07285 & 0.07168 & 307.9 \\
\hline 9 & 0.05965 & 0.05674 & 297.1 & 24 & 0.08150 & 0.07662 & 309.6 \\
\hline 10 & 0.06895 & 0.05916 & 299.2 & 25 & 0.08575 & 0.07537 & 309.6 \\
\hline 11 & 0.06545 & 0.05915 & 299.2 & 26 & 0.07800 & 0.07543 & 309.6 \\
\hline 12 & 0.06135 & 0.05906 & 299.2 & 27 & 0.07995 & 0.08016 & 311.5 \\
\hline 13 & 0.07010 & 0.06232 & 301.1 & 28 & 0.09125 & 0.08000 & 311.5 \\
\hline 14 & 0.07085 & 0.06223 & 301.1 & 29 & 0.08220 & 0.08001 & 311.5 \\
\hline 15 & 0.06425 & 0.06242 & 301.1 & - & - & - & - \\
\hline \multicolumn{8}{|c|}{$\left.\ln x_{2}=4.26 \pm 0.21-\cdot(2118 \pm 63) / T\right)$} \\
\hline \multicolumn{8}{|c|}{ 5-(3-nitro phenyl)-furyl-2-oxime } \\
\hline \multicolumn{8}{|c|}{ Propan-2-ol } \\
\hline 1 & 0.00355 & 0.00249 & 305.0 & 13 & 0.00615 & 0.00401 & 315.0 \\
\hline 2 & 0.00420 & 0.00264 & 307.0 & 14 & 0.00550 & 0.00404 & 315.0 \\
\hline 3 & 0.00370 & 0.00254 & 307.0 & 15 & 0.00690 & 0.00456 & 317.1 \\
\hline 4 & 0.00445 & 0.00300 & 308.9 & 16 & 0.00705 & 0.00503 & 319.5 \\
\hline 5 & 0.00435 & 0.00296 & 308.9 & 17 & 0.00710 & 0.00478 & 319.5 \\
\hline 6 & 0.00420 & 0.00293 & 308.9 & 18 & 0.00670 & 0.00479 & 319.5 \\
\hline 7 & 0.00460 & 0.00315 & 310.6 & 19 & 0.00765 & 0.00521 & 321.6 \\
\hline 8 & 0.00480 & 0.00326 & 310.6 & 20 & 0.00765 & 0.00530 & 321.6 \\
\hline 9 & 0.00455 & 0.00323 & 310.6 & 21 & 0.00750 & 0.00526 & 321.6 \\
\hline 10 & 0.00540 & 0.00351 & 313.1 & 22 & 0.00860 & 0.00598 & 323.6 \\
\hline 11 & 0.00510 & 0.00356 & 313.1 & 23 & 0.00725 & 0.00581 & 323.6 \\
\hline 12 & 0.00590 & 0.00401 & 315.0 & 24 & 0.00945 & 0.00576 & 323.6 \\
\hline \multicolumn{8}{|c|}{$\left.\ln x_{2}=9.28 \pm 0.55-\cdot(4666 \pm 173) / T\right)$} \\
\hline \multicolumn{8}{|c|}{ Acetonitrile } \\
\hline 1 & 0.00565 & 0.00252 & 302.5 & 15 & 0.00735 & 0.00359 & 310.9 \\
\hline 2 & 0.00535 & 0.00249 & 302.5 & 16 & 0.01025 & 0.00430 & 315.0 \\
\hline 3 & 0.00520 & 0.00247 & 302.5 & 17 & 0.00870 & 0.00437 & 315.0 \\
\hline 4 & 0.00640 & 0.00276 & 304.6 & 18 & 0.00845 & 0.00429 & 315.0 \\
\hline 5 & 0.00585 & 0.00274 & 304.6 & 19 & 0.01030 & 0.00479 & 317.1 \\
\hline 6 & 0.00565 & 0.00269 & 304.6 & 20 & 0.00975 & 0.00471 & 317.1 \\
\hline 7 & 0.00670 & 0.00304 & 306.5 & 21 & 0.01005 & 0.00481 & 317.1 \\
\hline 8 & 0.00645 & 0.00303 & 306.5 & 22 & 0.01095 & 0.00511 & 319.3 \\
\hline 9 & 0.00630 & 0.00298 & 306.5 & 23 & 0.01100 & 0.00510 & 319.3 \\
\hline 10 & 0.00745 & 0.00333 & 308.8 & 24 & 0.01120 & 0.00507 & 319.3 \\
\hline 11 & 0.00690 & 0.00332 & 308.8 & 25 & 0.01195 & 0.00561 & 321.6 \\
\hline 12 & 0.00710 & 0.00332 & 308.8 & 26 & 0.01225 & 0.00561 & 321.6 \\
\hline 13 & 0.00830 & 0.00366 & 310.9 & 27 & 0.01205 & 0.00566 & 321.6 \\
\hline 14 & 0.00770 & 0.00360 & 310.9 & - & - & - & - \\
\hline \multicolumn{8}{|c|}{$\left.\ln x_{2}=7.73 \pm 0.22-\cdot(4150 \pm 67) / T\right)$} \\
\hline \multicolumn{8}{|c|}{ Ethyl acetate } \\
\hline 1 & 0.00815 & 0.00707 & 298.1 & 23 & 0.01410 & 0.01187 & 315.8 \\
\hline 2 & 0.00810 & 0.00722 & 298.1 & 24 & 0.01320 & 0.01208 & 315.8 \\
\hline 3 & 0.00950 & 0.00780 & 300.6 & 25 & 0.01310 & 0.01175 & 315.8 \\
\hline
\end{tabular}




\begin{tabular}{|c|c|c|c|c|c|c|c|}
\hline 4 & 0.00850 & 0.00746 & 300.6 & 26 & 0.01555 & 0.01282 & 318.0 \\
\hline 5 & 0.00860 & 0.00779 & 300.6 & 27 & 0.01435 & 0.01285 & 318.0 \\
\hline 6 & 0.01145 & 0.00808 & 302.7 & 28 & 0.01405 & 0.01277 & 318.0 \\
\hline 7 & 0.00775 & 0.00788 & 302.7 & 29 & 0.01670 & 0.01335 & 319.6 \\
\hline 8 & 0.00880 & 0.00810 & 302.7 & 30 & 0.01415 & 0.01320 & 319.6 \\
\hline 9 & 0.01070 & 0.00876 & 304.6 & 31 & 0.01430 & 0.01327 & 319.6 \\
\hline 10 & 0.00955 & 0.00854 & 304.6 & 32 & 0.01725 & 0.01388 & 321.6 \\
\hline 11 & 0.00950 & 0.00863 & 304.6 & 33 & 0.01505 & 0.01363 & 321.6 \\
\hline 12 & 0.01125 & 0.00930 & 306.6 & 34 & 0.01560 & 0.01399 & 321.6 \\
\hline 13 & 0.01055 & 0.00926 & 306.6 & 35 & 0.01680 & 0.01524 & 323.6 \\
\hline 14 & 0.00990 & 0.00907 & 306.6 & 36 & 0.01780 & 0.01509 & 323.6 \\
\hline 15 & 0.01195 & 0.00985 & 308.9 & 37 & 0.01635 & 0.01512 & 323.6 \\
\hline 16 & 0.01130 & 0.00967 & 308.9 & 38 & 0.01810 & 0.01538 & 325.6 \\
\hline 17 & 0.01100 & 0.00986 & 308.9 & 39 & 0.01700 & 0.01512 & 325.6 \\
\hline 18 & 0.01240 & 0.01058 & 311.4 & 40 & 0.01645 & 0.01538 & 325.6 \\
\hline 19 & 0.01160 & 0.01051 & 311.4 & 41 & 0.01850 & 0.01652 & 327.4 \\
\hline 20 & 0.01375 & 0.01140 & 313.7 & 42 & 0.01865 & 0.01638 & 327.4 \\
\hline 21 & 0.01245 & 0.01123 & 313.7 & 43 & 0.01780 & 0.01652 & 327.4 \\
\hline 22 & 0.01260 & 0.01100 & 313.7 & - & - & - & - \\
\hline \multicolumn{8}{|c|}{$\left.\ln x_{2}=4.41 \pm 0.17-\cdot(2792 \pm 52) / T\right)$} \\
\hline \multicolumn{8}{|c|}{ 5-(4-nitro phenyl)-furyl-2-oxime } \\
\hline \multicolumn{8}{|c|}{ Propan-2-ol } \\
\hline 1 & 0.00365 & 0.00261 & 314.6 & 24 & 0.00720 & 0.00492 & 333.1 \\
\hline 2 & 0.00300 & 0.00257 & 314.6 & 25 & 0.00640 & 0.00474 & 333.1 \\
\hline 3 & 0.00255 & 0.00256 & 314.6 & 26 & 0.00710 & 0.00484 & 333.1 \\
\hline 4 & 0.00365 & 0.00258 & 316.7 & 27 & 0.00200 & 0.00143 & 300.9 \\
\hline 5 & 0.00375 & 0.00267 & 316.7 & 28 & 0.00230 & 0.00156 & 303.2 \\
\hline 6 & 0.00450 & 0.00299 & 318.5 & 29 & 0.00225 & 0.00160 & 303.2 \\
\hline 7 & 0.00425 & 0.00299 & 318.5 & 30 & 0.00205 & 0.00162 & 303.2 \\
\hline 8 & 0.00405 & 0.00292 & 318.5 & 31 & 0.00260 & 0.00172 & 306.2 \\
\hline 9 & 0.00365 & 0.00310 & 320.6 & 32 & 0.00280 & 0.00185 & 306.2 \\
\hline 10 & 0.00475 & 0.00316 & 322.3 & 33 & 0.00235 & 0.00172 & 306.2 \\
\hline 11 & 0.00470 & 0.00317 & 322.3 & 34 & 0.00285 & 0.00190 & 308.7 \\
\hline 12 & 0.00520 & 0.00353 & 324.1 & 35 & 0.00595 & 0.00398 & 326.9 \\
\hline 13 & 0.00540 & 0.00358 & 324.1 & 36 & 0.00580 & 0.00391 & 326.9 \\
\hline 14 & 0.00480 & 0.00355 & 324.1 & 37 & 0.00545 & 0.00403 & 326.9 \\
\hline 15 & 0.00515 & 0.00368 & 326.1 & 38 & 0.00625 & 0.00452 & 330.0 \\
\hline 16 & 0.00570 & 0.00369 & 326.1 & 39 & 0.00350 & 0.00447 & 330.0 \\
\hline 17 & 0.00475 & 0.00361 & 326.1 & 40 & 0.00770 & 0.00525 & 333.9 \\
\hline 18 & 0.00600 & 0.00415 & 328.3 & 41 & 0.00730 & 0.00522 & 333.9 \\
\hline 19 & 0.00600 & 0.00427 & 328.3 & 42 & 0.00735 & 0.00508 & 333.9 \\
\hline 20 & 0.00595 & 0.00424 & 328.3 & 43 & 0.00870 & 0.00555 & 336.3 \\
\hline 21 & 0.00670 & 0.00445 & 330.9 & 44 & 0.00790 & 0.00567 & 336.3 \\
\hline 22 & 0.00635 & 0.00448 & 330.9 & 45 & 0.00750 & 0.00551 & 336.3 \\
\hline 23 & 0.00615 & 0.00438 & 330.9 & - & - & - & - \\
\hline \multicolumn{8}{|c|}{$\left.\ln x_{2}=6.23 \pm 0.28-\cdot(3846 \pm 89) / T\right)$} \\
\hline \multicolumn{8}{|c|}{ Acetonitrile } \\
\hline 1 & 0.00945 & 0.00406 & 294.9 & 20 & 0.01265 & 0.00692 & 312.1 \\
\hline 2 & 0.00850 & 0.00404 & 294.9 & 21 & 0.01640 & 0.00734 & 314.3 \\
\hline 3 & 0.00865 & 0.00404 & 294.9 & 22 & 0.01560 & 0.00734 & 314.3 \\
\hline
\end{tabular}




\begin{tabular}{|c|c|c|c|c|c|c|c|}
\hline 4 & 0.00995 & 0.00424 & 297.1 & 23 & 0.01445 & 0.00720 & 314.3 \\
\hline 5 & 0.00860 & 0.00427 & 297.1 & 24 & 0.01740 & 0.00781 & 316.1 \\
\hline 6 & 0.00915 & 0.00427 & 297.1 & 25 & 0.01635 & 0.00769 & 316.1 \\
\hline 7 & 0.01000 & 0.00450 & 299.6 & 26 & 0.01615 & 0.00781 & 316.1 \\
\hline 8 & 0.00955 & 0.00446 & 299.6 & 27 & 0.01980 & 0.00842 & 318.2 \\
\hline 9 & 0.00955 & 0.00466 & 299.6 & 28 & 0.01665 & 0.00841 & 318.2 \\
\hline 10 & 0.00995 & 0.00471 & 301.5 & 29 & 0.01715 & 0.00841 & 318.2 \\
\hline 11 & 0.01030 & 0.00478 & 301.5 & 30 & 0.01940 & 0.00864 & 321.4 \\
\hline 12 & 0.00995 & 0.00473 & 301.5 & 31 & 0.01865 & 0.00878 & 321.4 \\
\hline 13 & 0.01230 & 0.00601 & 308.1 & 32 & 0.01725 & 0.00860 & 321.4 \\
\hline 14 & 0.01245 & 0.00599 & 308.1 & 33 & 0.02180 & 0.00925 & 323.1 \\
\hline 15 & 0.00980 & 0.00646 & 309.8 & 34 & 0.01805 & 0.00920 & 323.1 \\
\hline 16 & 0.01380 & 0.00651 & 309.8 & 35 & 0.01825 & 0.00925 & 323.1 \\
\hline 17 & 0.01310 & 0.00648 & 309.8 & 36 & 0.02140 & 0.01000 & 325.1 \\
\hline 18 & 0.01685 & 0.00693 & 312.1 & 37 & 0.02020 & 0.00990 & 325.1 \\
\hline 19 & 0.01450 & 0.00689 & 312.1 & 38 & 0.02035 & 0.00995 & 325.1 \\
\hline \multicolumn{8}{|c|}{$\left.\ln x_{2}=4.36 \pm 0.22-\cdot(2919 \pm 69) / T\right)$} \\
\hline \multicolumn{8}{|c|}{ Ethyl acetate } \\
\hline 1 & 0.01075 & 0.00904 & 296.4 & 13 & 0.01230 & 0.01091 & 304.6 \\
\hline 2 & 0.01025 & 0.00900 & 296.4 & 14 & 0.01215 & 0.01091 & 304.6 \\
\hline 3 & 0.01020 & 0.00918 & 296.4 & 15 & 0.01365 & 0.01154 & 306.7 \\
\hline 4 & 0.01135 & 0.00954 & 298.5 & 16 & 0.01380 & 0.01149 & 306.7 \\
\hline 5 & 0.01055 & 0.00956 & 298.5 & 17 & 0.01230 & 0.01154 & 306.7 \\
\hline 6 & 0.01050 & 0.00961 & 298.5 & 18 & 0.01380 & 0.01202 & 308.7 \\
\hline 7 & 0.01170 & 0.00985 & 300.6 & 19 & 0.01415 & 0.01195 & 308.7 \\
\hline 8 & 0.01100 & 0.00984 & 300.6 & 20 & 0.01320 & 0.01208 & 308.7 \\
\hline 9 & 0.01170 & 0.01009 & 300.6 & 21 & 0.01410 & 0.01243 & 310.6 \\
\hline 10 & 0.01235 & 0.01036 & 302.6 & 22 & 0.01415 & 0.01253 & 310.6 \\
\hline 11 & 0.01180 & 0.01051 & 302.6 & 23 & 0.01355 & 0.01231 & 310.6 \\
\hline 12 & 0.01385 & 0.01103 & 304.6 & - & - & - & - \\
\hline \multicolumn{8}{|c|}{$\left.\ln x_{2}=2.28 \pm 0.23-\cdot(2070 \pm 70) / T\right)$} \\
\hline
\end{tabular}

Graphical interpretations of the temperature dependences of solubility for the investigated compounds in chosen solutes ( 1 - propan-2-ol, 2 - acetonitrile, 3 - ethyl acetate) are shown in figures 3,4 and 5 .

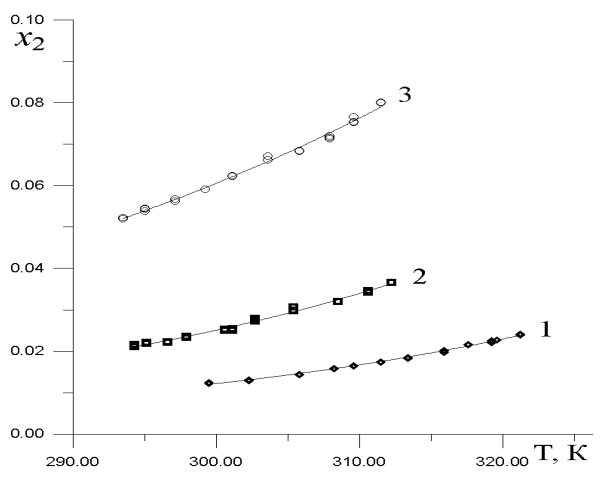

Figure 3. Temperature dependences of solubility for 5-(2-nitro phenyl)-2-furyl-oxime.

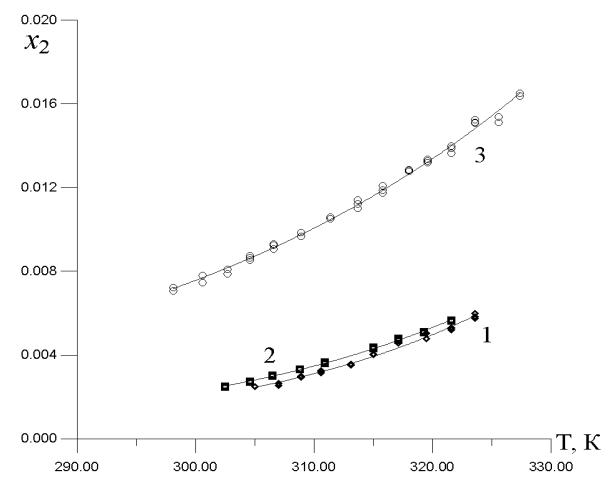

Figure 4. Temperature dependences of solubility for 5-(3-nitro phenyl)-2-furyl-oxime. 


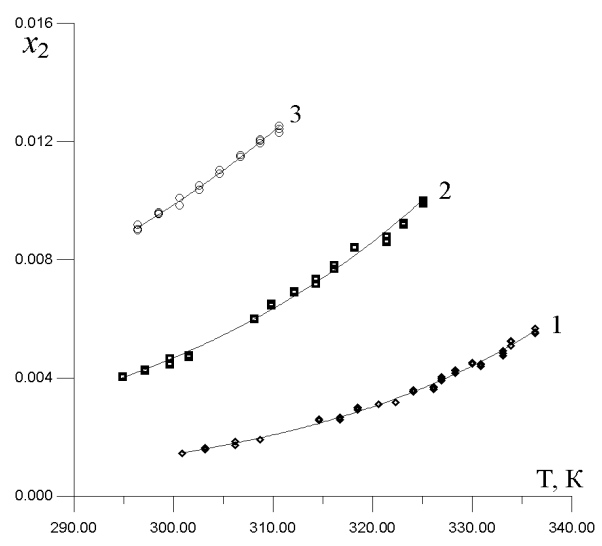

Figure 5. Temperature dependences of solubility for 5-(4-nitro phenyl)-2-furyl-oxime.

Enthalpies $\left(\Delta_{\text {sol }} H^{I / S}\right)$ and entropies $\left(\Delta_{\text {sol }} S^{I / S}\right)$ of dissolution were calculated from the temperature dependence of the solubility of these substances in solvents using equation 1 :

$$
\ln x_{2}=\Delta_{s o l} S^{I / S} / R ? \Delta_{\text {sol }} H^{I / S} /(R T)
$$

The results of calculations and the average temperatures of the studies range $T_{\text {sol }}$ are listed in Table 3 .

Table 3. Enthalpies and entropies of dissolution of the investigated compounds

\begin{tabular}{|c|c|c|c|}
\hline Solvent & $\boldsymbol{\Delta}_{\text {sol }} \boldsymbol{H}^{I / S}, \mathbf{k J} / \mathbf{m o l}$ & $\boldsymbol{\Delta}_{\text {sol }} \boldsymbol{S}^{I / S}, \mathbf{J} / \mathbf{m o l} \cdot \mathbf{K}$ & $\boldsymbol{T}_{\text {sol }}, \mathbf{K}$ \\
\hline \multicolumn{4}{|c|}{ 5-(2-nitro phenyl)-furyl-2-oxime } \\
\hline Propan-2-ol & $25.36 \pm 0.64$ & $47.8 \pm 2.1$ & 310.4 \\
\hline Acetonitrile & $23.19 \pm 0.84$ & $46.6 \pm 2.8$ & 303.3 \\
\hline Ethyl acetate & $17.61 \pm 0.53$ & $35.4 \pm 1.8$ & 302.5 \\
\hline \multicolumn{4}{|c|}{5 -(3-nitro phenyl)-furyl-2-oxime } \\
\hline Propan-2-ol & $38.8 \pm 1.4$ & $77.2 \pm 4.6$ & 313.1 \\
\hline Acetonitrile & $34.50 \pm 0.56$ & $64.3 \pm 1.8$ & 312.1 \\
\hline Ethyl acetate & $23.22 \pm 0.43$ & $36.7 \pm 1.4$ & 312.8 \\
\hline \multicolumn{4}{|c|}{5 -(4-nitro phenyl)-furyl-2-oxime } \\
\hline Propan-2-ol & $31.97 \pm 0.74$ & $51.8 \pm 2.3$ & 318.6 \\
\hline Acetonitrile & $24.27 \pm 0.57$ & $36.3 \pm 1.8$ & 310.0 \\
\hline Ethyl acetate & $17.21 \pm 0.58$ & $19.0 \pm 1.9$ & 303.5 \\
\hline
\end{tabular}

Temperature dependences of saturated vapor pressures and sublimation enthalpies were determined using integral effusion Knudsen method. Features of the installation, construction of cameras and membranes and the methodology of experiments were chosen according to recommendations [10].

Vacuum system of the installation reached the pressure of $0.1 \mathrm{~Pa}$ during $20 \pm 15$ seconds during the experiment. Mass of the compound, evaporated during experiment $(\Delta m)$ was determined from the mass difference of the camera, weighted before and after the experiment using VLR-20 scales with accuracy of $\pm 0.00005 \mathrm{~g}$. The accuracies of sample temperature $(T)$ and effusion time $(\tau)$ were $\pm 0.5 \mathrm{~K}$ and $\pm 5 \mathrm{~s}$ respectively.

The reliability of the effusion installation was confirmed in a series of experiments with benchmark benzoic acid K-1 (content of main component 99.95\%) in temperature range 322.7-354.1 K using a set of three membranes with orifice diameters $2.05,2.10$ and $2.15 \mathrm{~mm}$ respectively, and thickness $0.09 \mathrm{~mm}$. Results of experimental determination of temperature dependence of saturated vapor pressure for benzoic acid were processed using the least squares method and approximated in a linear equation:

$$
\ln P(P a)=(33.72 \pm 1.81)-(10727 \pm 619) \cdot 1 / T ; R=0.9849
$$


Sublimation enthalpy of benzoic acid, calculated from the equation, is equal to $89.2 \pm 5.1 \mathrm{~kJ} / \mathrm{mol}$ $\left(T_{\text {sub }}=343 \mathrm{~K}\right)$ and is in good accordance with the recommended value: $90.1 \pm 0.6 \mathrm{~kJ} / \mathrm{mol}\left(T_{\text {sub }}=353 \mathrm{~K}\right)$ from [11].

Methodology of saturated vapor determination for the investigated compounds is similar to the one for benzoic acid. Volatile impurities that could distort the results were removed on the first stage of experiment (surface formation). This stage was completed when the rate of mass loss was constant $\pm 1 \%$ at the fixed temperature. Primary results of the effusion studies, saturated vapor pressure $P$ of the investigated compound and temperatures of the experiments $(T)$ are shown in table 4.

Table 4. Primary results of effusion studies

\begin{tabular}{|c|c|c|c|c|c|c|c|c|}
\hline \multirow{2}{*}{ № } & \multirow{2}{*}{$\mathbf{T}, \mathbf{K}$} & \multirow{2}{*}{$\tau, \mathrm{s}$} & \multicolumn{2}{|c|}{ First membrane } & \multicolumn{2}{|c|}{ Second membrane } & \multicolumn{2}{|c|}{ Third membrane } \\
\hline & & & $\Delta \mathrm{m}, \mathrm{g}$ & $\mathrm{P}, \mathrm{Pa}$ & $\Delta \mathrm{m}, \mathrm{g}$ & $\mathbf{P}, \mathbf{P a}$ & $\Delta \mathrm{m}, \mathrm{g}$ & $\mathrm{P}, \mathrm{Pa}$ \\
\hline \multicolumn{9}{|c|}{ 5-(2-nitro phenyl)-furyl-2-oxime } \\
\hline 1 & 387.1 & 7236 & 0.00555 & 0.3380 & 0.00575 & 0.3397 & 0.00610 & 0.3730 \\
\hline 2 & 388.2 & 3640 & 0.00315 & 0.3819 & 0.00340 & 0.3999 & 0.00350 & 0.4261 \\
\hline 3 & 396.6 & 3634 & 0.00710 & 0.8714 & 0.00760 & 0.9050 & - & - \\
\hline 4 & 397.1 & 3659 & 0.00705 & 0.8599 & 0.00875 & 1.035 & 0.00885 & 1.084 \\
\hline 5 & 377.4 & 10843 & 0.00355 & 0.1425 & 0.00450 & 0.1752 & 0.00395 & 0.1592 \\
\hline 6 & 378.9 & 10838 & 0.00440 & 0.1770 & 0.00465 & 0.1815 & 0.00480 & 0.1939 \\
\hline 7 & 381.3 & 10844 & 0.00655 & 0.2642 & 0.00635 & 0.2484 & - & - \\
\hline \multicolumn{9}{|c|}{$\ln P=(-13449 \cdot 1 / T \pm 921)+(33.8 \pm 2.4), \rho=0.9821$} \\
\hline \multicolumn{9}{|c|}{ 5-(3-nitro phenyl)-furyl-2-oxime } \\
\hline 1 & 402.5 & 7234 & 0.00477 & 0.2963 & 0.00500 & 0.3013 & 0.00490 & 0.3056 \\
\hline 2 & 405.4 & 7240 & 0.00679 & 0.4229 & 0.00740 & 0.4472 & 0.00665 & 0.4160 \\
\hline 3 & 406 & 7240 & 0.00771 & 0.4806 & 0.00790 & 0.4777 & 0.00790 & 0.4945 \\
\hline 4 & 408.7 & 7235 & 0.00860 & 0.5382 & 0.00925 & 0.5616 & 0.00940 & 0.5908 \\
\hline 5 & 410.2 & 7234 & 0.00835 & 0.5236 & 0.00845 & 0.5140 & 0.00815 & 0.5132 \\
\hline 6 & 419.8 & 7222 & 0.01920 & 1.220 & 0.01735 & 1.070 & 0.01775 & 1.133 \\
\hline 7 & 395.7 & 10822 & 0.00485 & 0.1997 & 0.00495 & 0.1977 & 0.00495 & 0.2046 \\
\hline 8 & 407.2 & 10824 & 0.00925 & 0.3862 & 0.00910 & 0.3686 & 0.00935 & 0.3921 \\
\hline 9 & 407.5 & 10825 & 0.01105 & 0.4615 & 0.01145 & 0.4639 & 0.01130 & 0.4740 \\
\hline $\begin{array}{l}1 \\
0\end{array}$ & 394.1 & 10828 & 0.00335 & 0.1376 & 0.00350 & 0.1394 & 0.00340 & 0.1402 \\
\hline $\begin{array}{l}1 \\
1\end{array}$ & 390 & 10827 & 0.00250 & 0.1021 & 0.00260 & 0.1030 & 0.00260 & 0.1067 \\
\hline \multicolumn{9}{|c|}{$\ln P=(-13109 \cdot 1 / T \pm 790)+(31.4 \pm 2.0), \rho=0.9736$} \\
\hline \multicolumn{9}{|c|}{ 5-(4-nitro phenyl)-furyl-2-oxime } \\
\hline 1 & 414.6 & 3619 & 0.00175 & 0.2205 & 0.00180 & 0.2200 & 0.00175 & 0.2215 \\
\hline 2 & 418 & 3619 & 0.00250 & 0.3163 & 0.00245 & 0.3007 & 0.00245 & 0.3113 \\
\hline 3 & 422.9 & 3696 & 0.00360 & 0.4486 & 0.00375 & 0.4534 & 0.00370 & 0.4630 \\
\hline 4 & 425.1 & 3622 & 0.00380 & 0.4845 & 0.00425 & 0.5257 & 0.00410 & 0.5249 \\
\hline 5 & 425.1 & 3626 & 0.00385 & 0.4903 & 0.00400 & 0.4942 & 0.00540 & 0.4988 \\
\hline 6 & 428.9 & 3625 & - & - & 0.00545 & 0.6765 & 0.00390 & 0.6939 \\
\hline
\end{tabular}

Graphical interpretation of temperature dependences of saturated vapor pressures for investigated compounds is shown in figure 6 .

Values of sublimation enthalpies $\left(\Delta_{c r}^{g} H\right)$ and entropies $\left(\Delta_{c r}^{g} S\right)$, calculated from the linear form of Clapeyron-Clausius equation (2) of temperature dependence of saturated vapor pressure as well as average temperatures $T_{\text {sub }}$ are listed in table 5 . 


$$
\ln P=\Delta_{c r}^{g} S / R-\Delta_{c r}^{g} H /(R \cdot T)
$$

Table 5. Sublimation enthalpies and entropies of the investigated compounds

\begin{tabular}{|c|c|c|c|}
\hline Compound & $\Delta_{c r}^{g} \boldsymbol{H}, \mathbf{k J} / \mathbf{m o l}$ & $\Delta_{c r}^{g} \boldsymbol{S}, \mathbf{J} / \mathbf{m o l} \cdot \mathbf{K}$ & $\boldsymbol{T}_{\text {sub }}, \mathbf{K}$ \\
\hline $\mathrm{A}$ & $111.8 \pm 7.7$ & $185 \pm 20$ & 387.3 \\
\hline $\mathrm{B}$ & $109.0 \pm 6.6$ & $165 \pm 16$ & 404.9 \\
\hline $\mathrm{C}$ & $113.6 \pm 6.6$ & $166 \pm 16$ & 421.8 \\
\hline
\end{tabular}

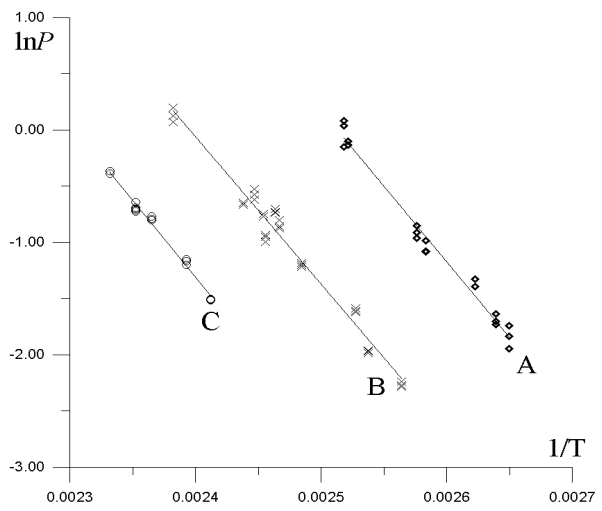

Figure 6. Temperature dependences of saturated vapor pressures for investigated compounds.

\section{Discussion}

Sublimation and dissolution enthalpies and entropies were determined in different temperature ranges conditioned by the properties of compounds and solvents. $\Delta_{c r}^{g} H$ and $\Delta_{c r}^{g} S$ needed to be adjusted to the average temperatures of solubility studies. Heat capacity changes $\Delta C_{p}$ during sublimation were crucial for these calculations. We couldn't determine these values experimentally, so the half-empiric method [12] was used for the purpose:

$$
\begin{gathered}
\Delta_{c r}^{g} H_{T s o l}=\Delta_{c r}^{g} H_{T s u b}+(0.259 \pm 0.041) \cdot M \cdot\left(T_{s u b}-T_{s o l}\right) \\
\Delta_{c r}^{g} S_{T s o l}=\Delta_{c r}^{g} S_{T s u b}+(0.259 \pm 0.041) \cdot \operatorname{In} \frac{T_{s u b}}{T_{s o l}}
\end{gathered}
$$

where M - molecular weight of the compound $(\mathrm{g} / \mathrm{mol}), T_{s u b}$ - average temperature of vapor pressure studies, $T_{\text {sol }}$ - average temperature of the solubility studies.

Solution enthalpy $\Delta_{s o l} H^{I / S}$ is the enthalpy of individual solute's $I$ transfer from its standard state (solid for the investigated compounds) to solution in solvent $S$. Solvation enthalpy $\Delta_{\text {solvat }} H^{I / S}$ is the enthalpy of solute's $I$ isothermal transfer from the ideal gas state to solution in solvent $S$. Sublimation enthalpy $\Delta_{c r}^{g} H$ is the enthalpy of solute I transfer from the solid state to the ideal gas state. The above listed properties follow the equation [9]:

$$
\begin{gathered}
\Delta_{\text {sol }} H_{T \text { sol }}^{I / S}=\Delta_{c r}^{g} H_{T s o l}+\Delta_{\text {solvat }} H_{T \text { Tsol }}^{I / S} \\
\Delta_{\text {sol }} S_{T \text { sol }}^{I / S}=\Delta_{c r}^{g} S_{T s o l}+\Delta_{\text {solvat }} S_{T \text { Tsol }}^{I / S}
\end{gathered}
$$

The thermodynamic parameters of solvation are listed in table 6. Physical properties of the used solvents are listed in table 7 .

In the row of solvents propan-2-ol - acetonitrile - ethyl acetate acceptor number $\mathrm{A}_{\mathrm{N}}$, which describes the solvents protonation ability, is decreasing (table 7), and the chemical consistency, which is characterized by free Gibbs energy (table 6) is increasing for all the studied systems. Oximes are likely to form intermolecular hydrogen bonds $\mathrm{N}$. · HO. The more protonation ability of the solvent is, the 
more these bonds it can destroy. Solvents with lesser $A_{N}$ are of advisable use in chemical and technological processes involving these compounds.

Table 6. Thermodynamic properties of solvation for investigated compounds at $\mathrm{T}_{\mathrm{sol}}$

\begin{tabular}{|c|c|c|c|c|}
\hline Solvent & $T_{\text {sol }}, K$ & $\Delta_{\text {solvat }} \boldsymbol{H}_{\text {Tsol }}^{I / S}$ & $\Delta_{\text {solvat }} S_{T \text { Isol }}^{I / S}$ & $\Delta_{\text {solvat }} G_{T \text { sol }}^{I / S}$ \\
\hline \multicolumn{5}{|c|}{ 5-(2-nitro phenyl)-furyl-2-oxime } \\
\hline Propan-2-ol & 310.4 & $-91.0 \pm 7.7$ & $-151 \pm 20$ & $-44.3 \pm 11$ \\
\hline Acetonitrile & 303.3 & $-93.7 \pm 7.7$ & $-153 \pm 20$ & $-47.2 \pm 11$ \\
\hline Ethyl acetate & 302.5 & $-99.3 \pm 7.7$ & $-165 \pm 20$ & $-49.5 \pm 11$ \\
\hline \multicolumn{5}{|c|}{ 5-(3-nitro phenyl)-furyl-2-oxime } \\
\hline Propan-2-ol & 313.1 & $-75.7 \pm 6.7$ & $-104 \pm 17$ & $-43.3 \pm 9.5$ \\
\hline Acetonitrile & 312.1 & $-80.1 \pm 6.6$ & $-117 \pm 16$ & $-43.7 \pm 9.3$ \\
\hline Ethyl acetate & 312.8 & $-91.2 \pm 6.6$ & $-144 \pm 16$ & $-46.2 \pm 9.3$ \\
\hline \multicolumn{5}{|c|}{ 5-(4-nitro phenyl)-furyl-2-oxime } \\
\hline Propan-2-ol & 318.6 & $-87.8 \pm 6.6$ & $-131 \pm 16$ & $-46.0 \pm 9.5$ \\
\hline Acetonitrile & 310.0 & $-96.0 \pm 6.6$ & $-148 \pm 16$ & $-50.0 \pm 9.5$ \\
\hline Ethyl acetate & 303.5 & $-103.5 \pm 6.6$ & $-167 \pm 16$ & $-52.9 \pm 9.5$ \\
\hline
\end{tabular}

Table 7. Physical properties of the used organic solvents

\begin{tabular}{|c|c|c|c|c|c|c|}
\hline Solvent & $\boldsymbol{T}_{\text {boil }}, \mathbf{K}$ & $n_{D}^{20}$ & $\boldsymbol{\varepsilon}$ & $\boldsymbol{\mu}, \mathbf{D}$ & $\mathbf{A}_{\mathbf{N}}$ & $\mathbf{D}_{\mathbf{N}}$ \\
\hline Propan-2-ol & 355.4 & 1.3774 & 19.9 & 1.68 & 22.3 & 20.0 \\
\hline Acetonitrile & 354.6 & 1.3463 & 37.5 & 3.5 & 18.9 & 14.1 \\
\hline Ethyl acetate & 350.0 & 1.3724 & 6.02 & 1.85 & 9.3 & 17.1 \\
\hline
\end{tabular}

The compensational effect between solvation enthalpies and entropies (figure 7), which depends on the reconstruction of intermolecular interactions between the solvents' and solutes' molecules, is also present in all of the investigated systems.

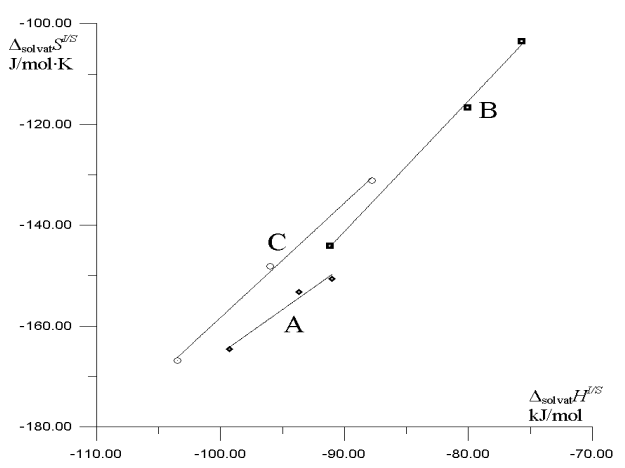

Figure 7. The compensational effect between solvation enthalpies and entropies.

Perhaps, it is caused by the partial destruction of hydrogen bonds of solute-solute and solvent-solvent types and the formation of new bonds of solvent-solute type. The larger number of investigated oximesolvent systems is needed for the deeper explanation of this phenomenon.

\section{Conclusions}

The temperature dependences of saturated vapor pressures and temperature dependences of solubility for nitro phenyl derivatives of furfural oxime are well approximated by the linear forms of ClapeyronClausius (eq. 2) and Schroeder's (eq. 1) equations, respectively. Sublimation and dissolution enthalpies and entropies were reliably calculated using these equations. Solvation enthalpies entropies and Gibbs 
energies were estimated from the obtained data. The presence of compensational effect between solvation enthalpies and entropies in the investigated systems was established. The increase of solubility with decrease of solvent's acceptor number is shown. From the chosen solvents ethyl acetate has the highest chemical consistency with oximes.

\section{References}

1. A. Datta, S. Walia, B.S. Parmar "Some Furfural Derivatives as Nitrification Inhibitors." J. Agric. Food Chem., vol. 49, pp. 4726-4731, 2001.

2. R.I. Meltzer, A.D. Lewis, J.A. King "Antitubercular Substances. IV. Thioamides.", J. Am. Chem. Soc., vol. 77, no. 15 , pp. 4062-4066, 1955.

3. O.P. Pandey, S.K. Sengupta, R.Chandra "Efficacy of organophosphorous derivatives containing oximes against fungal pathogens of sugarcane." EJEAFChe, vol. 5, no. 5, pp. 1515-1521, 2006.

4. D.J.Ager "The synthesis of 2-, 5-distributed furans." Tetrahedron Letters, vol. 24, no. 49, pp. 5441-5444, 1983.

5. Bessin P., Laforest J., Thuillier G., US Pat. No. 4207319; Chem. Abstr., 93, 220574 (1980).

6. Thuillier G., Laforest J., Bessin P., Ger. Pat. No. 2449205; Chem. Abstr., 83, 97004 (1975).

7. Laforest J., Ger. Pat. No. 2922799; Chem. Abstr., 93, 180989; Chem. Abstr., 92, 180989 (1980).

8. Brouwer W.G., Canadian Patent No. 2163175; Chem. Abstr., 125, 221559 (1996).

9. B. Solomonov, A. Varfolomeev, R. Nagrimanov, V. Novikov, A. Buryurov, Yu. Fedorova, T. Mukhametzyanov "New method for determination of vaporization and sublimation enthalpy of aromatic compounds at 298.15K using solution calorimetry technique and group-additivity scheme." Thermochemica Acta, vol. 622, pp. 88-96, 2015.

10. Ribeiro da Silva A.V.M., Monte J.S.M. "The construction, testing and use of a new Knudsen effusion apparatus" Thermochemica Acta, vol. 171. pp. 169, 1990.

11. J.S. Chickos, W.E. Acree "Enthalpies of vaporization of organic and organometallic compounds 1880-2002", J. Phys. Chem.Ref. Data, vol. 32, no. 2, pp. 519-878, 2003.

12. Sobechko I.B., Van-Chin-Syan Yu.Ya, Kochubey V.V., Prokop R.T., Velychkivska N.I., Horak Yu.I., Dibrivnyi V.M, Obushak M.D. "Thermodynamic properties of furan-2-carboxylic and 3-(2-furyl)-propanoic acids"(in Russian), Journal of physical chemistry, vol. 88, no. 12, pp. 1885-1892, 2014. 